Monatsschrift f. Geburtshülfe u. Gynäkologie 1918;48:I-IV

\title{
Contents, Vol. 48, 1918
}

Inhalts-Verzeichnis.

Original-Arbeiten. Seite

Abraham, O., Versuche einer serologischen Geschlechts-

bestimmung 163

Ah If eld, P., Die Säuglingsverluste der ersten zehn

Lebenstage in der Marburger Frauenklinik, 1885 bis 190743 Bauch, B., Bevölkerungsproblem und Hebammen-Lehr-

anstalt 49

B 1 e y , K., Die Aborte in den Jahren 1912-17 einschließlich 393 Bracht, E., Über den Ersatz des. Blasenschließmuskels 411 E b e 1 e r , F., Zur Wirksamkeit des Dispargens bei puerperalen Erkrankungen 264

Trankl,O., Über das sogenannte Adenoma malign um der

Gebärmutter. (Hierzu Taf. I) 178

Gच e r m a n n , H., Zur Indikation der Fixationsmethoden des

Uterus mit einem ka suistischen Beitrag zu Bumms Colli-

fixatio uteri . 187

Guttmann, E., Über die Heizsondenbehandlung der

weiblichen Gonorrhoe $\quad 428$

Heyn, A., Zur Kasuistik der Bauchspalte 35

Hofbauer, J., Sieben Jahre Hypophysenmedikation in

der praktischen Geburtshülfe . 325

Ill n e r, Ein Fall von Schwangerschaft bei außergewöhnlich

engem Hymen 33

Kehrer, E., Soil das Vulvakarzinom operiert oder be-

strahlt werden ? (Hierzu Taf. II) 346

Koehler, H., Primäre Abdominalgravidität. ...... 83

Littauer, A·, Über 250 Fälle von Uterusfixation mittels

subfaszialer Eröffnung des Leistenkanals 420

Lönne, F., Schwangerschaft, Geburt und Wochenbett

Erstgebärender in Kriegs- und Friedenszeiten .... 9 Number ger,L., Erlebnisse der Münchener

Universitäts-

Frauenklinik mit der „spanischen Krankheit”233

Schröder, E., Entstehung und Vererbung von Miß-

bildungen an der Hand eines Hypodaktylie-Stammbaumes.

(Hierzu Taf. II-V) 210

Schroder, R., „Gallensteine” in einem Dermoid. Zur

Frage der Kugelbildung in Dermoidgeschwülsten ... 98 Schweitzer, B., Erfahrungen bei der hohen Sakral-

Anästhesie bei gynäkologischen Operationen 
S i e g r i s t , H., Vier Jahre Röntgenkastration im Frauenspital Basel 103

$-\mathrm{IV}-$

Eeite

Un terberger, F., Die spontane Ruptur des rudimentären

Nebenhorns iníolge einer Hämatometra 256

Werner, E., Die kombinierte intravenose und intra-

muskuläre Anwendung von Chinin als Wehenmittel . . 309

Zu den Tagesíragen. Zum Verhältnis der pathologisehen Anatomie zur klinischen

Medizin. Von 0. Lubarsch 294

Über das medizín $1 / 8$ che Frauenstudium in Deutsehland. Von

A. Mart in ...... 222

$\mathrm{Zu}$ den Bevölkerungsfragen .305

Die deutsehen Frauenkliniken im achten Kriegssemester . . 136

Beriehíe.

Die c $7 / 8$ iische geburtshü†'lioh-gynäkologische Literatur 1916 bis 1917. Von Dir. Dr. Albeek in Aarhus . . . .371

Die geburtshülflich-gynäkologischen Dissertationen der Deut sehen und Schweizer Universitäten (Sommer-Sem. 1917).

Von Prof. Dr. F. Weber in München .

273

Ignaz Philipp Semmelweis hundertster Geburtstag 1

S. Pozzi $\uparrow \quad 82$

Leopold Meyer $\dagger \quad 141$

$\mathrm{Ph}$. Jung $\dagger 158$

Anton R. v. Noga Mars $\uparrow . . .298$

Vereins- und Literaturbeilage.

Gesellschaft für Geburtshülfe und Gynäkologie in Berlin.

Sitzung vom 10. V., 14. VI., 21. VI., 26. IV. u. 25. X. 1918

70, 144, 227, 444 GynäkologischeGesellschaft in Breslau. Sitzung v. 30. IV. 191865

Gebnrfcshülflich-gynäkologische Gesellschaft in Wien. Sitzung

vom 16. IV., 14. V. u. U. VI.

145,300

Geijurtshülflich-gynäkologische Mitteilungen aus Medizini-

schen Gesellschaften und Vereinen 71, 150, 229, 304, 381, 445

Buchanzeigen 72, 151, 229, 382, 446

Literatur-Verzeichnis 79, 152, 232, 307, 391, 455

Beriehtigung 64

Personalien . 82, 156, 226, 380, 443

Saehregister 458

Namenregister 461 